\title{
Trafic Signs Detection Based On Saliency Map Using Canny Edge
}

\author{
Putri Alit Widyastuti Santiary ${ }^{1}$, I Made Oka Widyantara ${ }^{2}$, Rukmi Sari Hartati ${ }^{3}$ \\ ${ }^{1,2,3}$ Master of Information Systems and Computer, Electrical Engineering Graduate Program \\ Udayana University \\ Denpasar, Bali, Indonesia \\ ${ }^{1}$ putrialit@gmail.com \\ 2oka.widyantara@unud.ac.id \\ 3rukmisari@unud.ac.id
}

\begin{abstract}
This paper proposed Canny edge detection to detected saliency map on traffic sign. The edge detection functions by identifying the bounds from an object on an image. The edge of an image is an area that has a strong intensity of light.The pixel intensity of an image changes from low to high values or otherwise. Detecting the edge of an image significantly will decrease the amount of data and filters insignificant information by not deleting necessary structure from the image. The image used for this paper is a digital capture of a traffic sign with a background. The result of this study shows that Canny edge detection creates saliency map from the traffic sign and separates the road sign from the background. The image result tested by calculating the saliency distance between a tested image and trained image using normalized Euclidean distance. The value of normalized Euclidean distance is set between 0 to 2. The testing process is done by calculating the nearest distance between the tested vector features and trained vector features. From the examination as a whole, it can be concluded that road sign detection using saliency map model can be built by Canny edge detection. From the whole system examination, it resulted a accuracy value of 0,65 . This value shows that the data was correctly classified by $65 \%$. The precision value has an outcome of 0,64 , shows that the exact result of the classification is $64 \%$. The recall value has an outcome of 0,94 . This value shows that the success rate of recognizing a data from the whole data is $94 \%$.
\end{abstract}

Index Terms - traffic signs, saliency map, Canny edge detection, normalized Euclidean distance.

\section{INTRODUCTION}

The automatic traffic signs detection is an interesting topic on intelligent transportation systems and has been implemented on various applications such as driving safety and automatic vehicle guidance. Traffic signs can be in the form of symbols, letters, numbers, sentences or combinations and serves as warning, prohibition, command or directions for road users [9]. The problem of traffic sign recognition has some beneficial characteristics such as unique design of traffic sign board, which means the sign shape variations are small, and significantly color contrast against the environment [18].

Traffic sign detection by real time and accurate has become an attention in Computer vision practices. Computer vision aims to computerized human vision or create digital images from original images (based on human vision). The aim of traffic lights detection system is to guide and give directions to drivers while driving, thus increasing the road safeness. This system can be classified into two parts, which are a system that provides information to drivers and system that acts all at once for the vehicle. [8].

Traffic signs are designed to be salient from the objects around them or background. The computation methods to automatically detects those traffic signs in the image remains significant. Humans can identify salient areas in their visual fields with surprising speed and accuracy before performing actual recognition. Computationally detecting such salient image regions remains a significant goal, as it allows preferential allocation of computational resources in subsequent image analysis and synthesis [3]. The objective is to separates the desired objects from the background around the image. This study proposed Canny edge detection to obtains saliency map model of traffic signs detection.

\section{CANNY EDGE DETECTION}

The objective of edge detection is to identify the boundaries of an object inside image. The edges of an object are areas that has a strong light intensity. The pixel intensity of an object changes from low to high value or vice versa. 
Detecting the edges of an object significantly reduce the amount of data and filter out useless information without loss important of structure of the image. One of the modern edge detection algorithms is edge detection using the Canny method.

Canny edge detection detects the actual edges with minimum error rate. Canny operators are designed to produce optimal edge image output. The Canny edge detection algorithm as follows [15]:

1. Application of Gaussian filter to reduce noise.

This process produces an image that looks a bit blurry. It aims to get the edge of the real object. If not applied, the fine lines will also be detected as edges. Here is one example of a Gaussian filter with $\sigma=1.4$ below.

$$
\frac{1}{115}\left[\begin{array}{ccccc}
2 & 4 & 5 & 4 & 2 \\
4 & 9 & 12 & 9 & 4 \\
5 & 12 & 15 & 12 & 5 \\
4 & 9 & 12 & 9 & 4 \\
2 & 4 & 5 & 4 & 2
\end{array}\right]
$$

2. Edges value detection.

Finds the edge value by connecting the image gradient using a pair of $3 \times 3$ convolution matrices $G x$ and $G y$ below. $G x$ predicts the gradient value for $\mathrm{x}$ direction (column) and the other predicts the gradient value for the $\mathrm{y}$ direction (row).

$$
\left[\begin{array}{ccc}
-1 & 0 & +1 \\
-2 & 0 & +2 \\
-1 & 0 & +1
\end{array}\right] \quad\left[\begin{array}{ccc}
+1 & +2 & +1 \\
0 & 0 & 0 \\
-1 & -2 & +1
\end{array}\right]
$$

The edge gradient $G$ calculated by equation (1):

$$
G=G_{x}+G_{y}
$$

3. Edges direction detection.

Finds the edge direction $\theta$ using equation (2):

$$
\theta=\arctan \left(\frac{G_{y}}{G_{x}}\right)
$$

4. Edges direction connection.

The directions of the edges are connected in a direction that can be traced according to the original image. The following example uses a $5 \times 5$ pixel image.

\begin{tabular}{|c|c|c|c|c|}
\hline$x$ & $x$ & $x$ & $x$ & $x$ \\
\hline$x$ & $x$ & $x$ & $x$ & $x$ \\
\hline$x$ & $x$ & $a$ & $x$ & $x$ \\
\hline$x$ & $x$ & $x$ & $x$ & $x$ \\
\hline$x$ & $x$ & $x$ & $x$ & $x$ \\
\hline
\end{tabular}

In above matric, (a) pixel has four possible directions when looks into surrounding pixels. The directions are as follow: $0^{\circ}$ horizontal direction, $45^{\circ}$ positive diagonal direction, $90^{\circ}$ vertical direction, and $135^{\circ}$ negative diagonal direction. Hence, the edge should point into the four directions, depends on which direction is closest. For example, if the angle is $3^{\circ}$, its became $0^{\circ}$. The next step, divide into four colors so that the lines with different directions will have different colors. The divisions as follow: $\left(0^{\circ}-22.5^{\circ}\right)$ and $\left(157,5^{\circ}-180^{\circ}\right)$ is yellow, $\left(22.5^{\circ}-\right.$ $\left.67.5^{\circ}\right)$ is green, and $\left(67,5^{\circ}-157,5^{\circ}\right)$ is red.

5. Non-maximum suppression process.

Non-maximum suppression is used to trace edge within edge direction and filter out pixels unconsidered as edge. This will show a fine line on the output image.

6. Binary with applying two thresholds.

\section{RESEARCH METHODE}

The dataset used in this study are digital images of traffic signs from image dataset at URL

http://aganemon.tsc.uah.es/Investigacion/

gram/traffic_signs.html [1]. Dataset contains of 325 images and processed using Java programming language. Computer program runs on portable computer with dual-core T2080 processor $1.73 \mathrm{GHz} 1 \mathrm{~GB}$ memory. In Figure 1 below shows process flow diagram.

\section{A. Input Image}

The input- image has some characteristics that came from shooting conditions. Distance shooting made differences of close or long-distance nput-image. Lighting condition made input-image bright or dark. Shooting orientation showed that images are shoot at different orientations. Not all images at perpendicular position, there are some images shoot at a somewhat skewed position. 


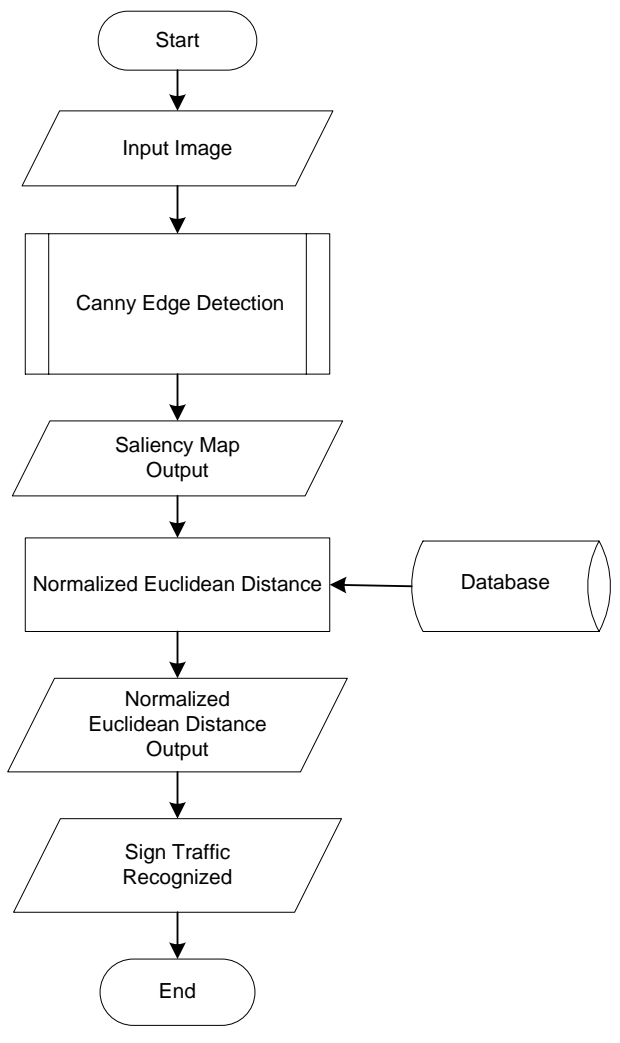

Figure 1. Proces Flow Diagram

\section{B. Canny Edge Detection}

Edge detection of traffic signs used Canny procedure. The edge of an object within image is an area that has a strong light intensity. Intensity of pixel changes from low to high value or vice versa. First, the original image in the r-g-b color space is converted into a grayscale image $I$ with equation (3) [16].

$I=(r+g+b) / 3$

Second, the grayscale image is filtered by applying a Gaussian filter with $\sigma=1.4$ as follows:

$$
\frac{1}{115}\left[\begin{array}{ccccc}
2 & 4 & 5 & 4 & 2 \\
4 & 9 & 12 & 9 & 4 \\
5 & 12 & 15 & 12 & 5 \\
4 & 9 & 12 & 9 & 4 \\
2 & 4 & 5 & 4 & 2
\end{array}\right]
$$

And then, the edge value is obtained by applying two matrices Gx and Gy as below:

$$
\left.\begin{array}{lll}
-1 & 0 & +1 \\
-2 & 0 & +2 \\
-1 & 0 & +1
\end{array}\right]
$$

$$
\left[\begin{array}{ccc}
+1 & +2 & +1 \\
0 & 0 & 0 \\
-1 & -2 & +1
\end{array}\right]
$$

\section{Threshold}

Binary image is obtained by applying a threshold. Binary process aims to adjusts intensity of gray level image. It changes image initially a 255-gray level ( 8 bit) into binary (black and white). Within threshold results become clear.

One thing to consider in threshold process is to chooses a threshold value. Pixels value below threshold value will be set as black otherwise as white. The threshold value $T$ is calculated by using equation (4) [15].

$T=\frac{f_{m a k s}+f_{\min }}{2}$

Images result of binary process is final result of saliency map.

\section{Normalized Euclidean distance}

Normalized Euclidean distance from cropped test-image results of saliency map compared with train-image dataset. The normalized Euclidean distance of two vector feature of $u$ and $v$ is shown by equation (5) [13].

$\bar{d}(u, v)=\left(\sum_{i}\left(\bar{u}_{i}-\bar{v}_{i}\right)^{2}\right)^{1 / 2}$

with,

$\bar{u}_{i}=\frac{u_{i}}{\|u\|}, \bar{v}_{i}=\frac{v_{i}}{\|v\|}$

$\|v\|=\left[\sum_{i} v_{i}^{2}\right]^{1 / 2}$

where:

$$
\begin{aligned}
& \bar{d}(u, v)=\text { Normalized Euclidean distance } \\
& u, v=\text { vector feature. }
\end{aligned}
$$

The smaller of score $\bar{d}(u, v)$, the more similar of two feature vectors or vice versa. Characteristic of normalized Euclidean distance is that the result within range of $0.0 \leq$ $\bar{d}(u, v) \leq 2.0$.

Output image of saliency map is tested by calculates the saliency distance between test-image and train-image in the database. The test results are classified into two types, namely signs and not-signs.

\section{RESULTS AND DISCUSSION}

The saliency map in this study has been tested from collection of digital images on dataset. The original image can be seen in Figure $2(a)$ through $(h)$ in which have differences type of test data.
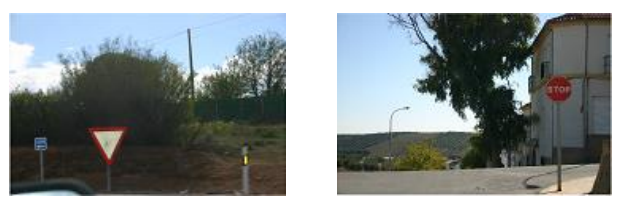
(a)

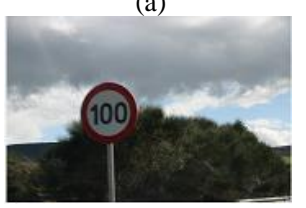

(c)

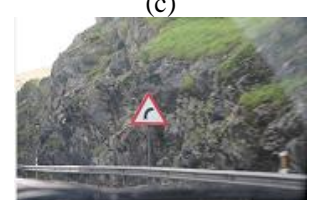

(e)

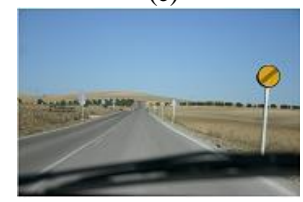

(g) (b)

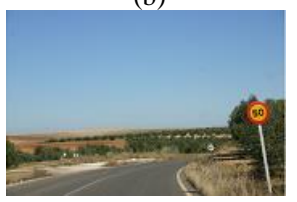

(d)

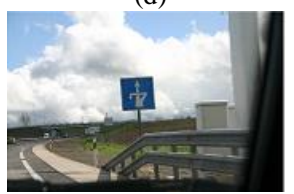

(f)

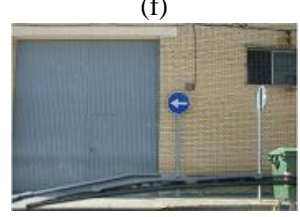

(h)

Figure 2. The original images (a) Data 1, (b) Data 2, (c) Data 3, (d) Data 4, (e) Data 5, (f) Data 6, (g) Data 7, (h) Data 8

Figure remark: Data 1 and 2 are priority signs, data 3 and 4 are prohibition signs, data 5 is a danger sign, data 6 is warning and information sign, data 7 is end of prohibition sign and data 8 is obligation sign.

Process stages of obtaining a saliency map for traffic signs using Canny edge detection for data 1 is shown by figure (3).

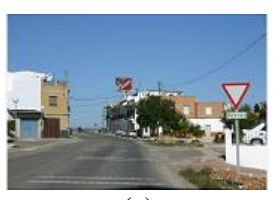

(a)

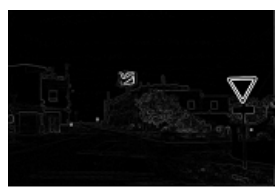

(d)

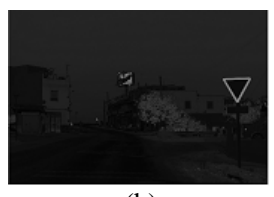

(b)

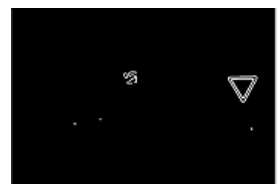

(e)

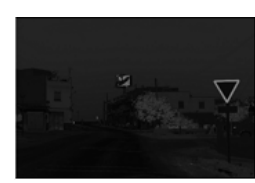

(c)

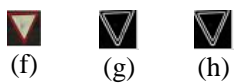

Figure 3. Saliency map process stages of traffic signs using Canny edge detection.

1) The Original Images: Traffic signs image with background in r-g-b color space. This is used as input data on the system. The original image is shown in Figure 3 (a).

2) Grayscale: Converts the r-g-b original images into one grayscale image. Each intensity values of color channel are summed up into a grayscale image $I$. And then obtains the absolute averaged value of $I$ by eliminates decimal values. Grayscale conversion produces a new different gray level image. The image has various level of black to white. Grayscale conversion results are shown in Figure 3 (b).

3) Gaussian filter: Applied a Gaussian filter to noise reduction. The grayscale image filtered with a Gaussian filter with $\sigma=1.4$. This process produces a bit blurry image. The Gaussian filter results are shown in Figure 3 (c).

4) Canny Edge Detection: Canny edge detection finds the edge value by connecting image gradient using a $3 \times 3$ convolution matrix pair. The result of Canny edge detection is shown in Figure 3 (d).

5) Binaryization: Binary images obtained by applying two thresholds. This is final stage to gets the saliency map of traffic signs image. The result of the binary process is shown in Fig. 3 (e).

6) Cropping: Cropping process for original image and saliency map image result. Cropping separates image object from its background. Cropped results used to calculate the normalized Euclidean distance between the test-data and the train-data. Figures 3 (f), 3 (g), and 3 (h). show cropped original image, test-data and comparison data. Cropped test-data results of the saliency map process. Comparative data are train-data, in which results of a saliency map processes that has been stored in database.

7) Normalized Euclidean Distance: For assessment of result normalized Euclidean distance of cropped saliency map image results (test-data matrix) compared with training data (train-data matrix) using equation (5). Test-data matrix of Data 1 can be seen in figure 4 and figure 5 for train-data matrix.

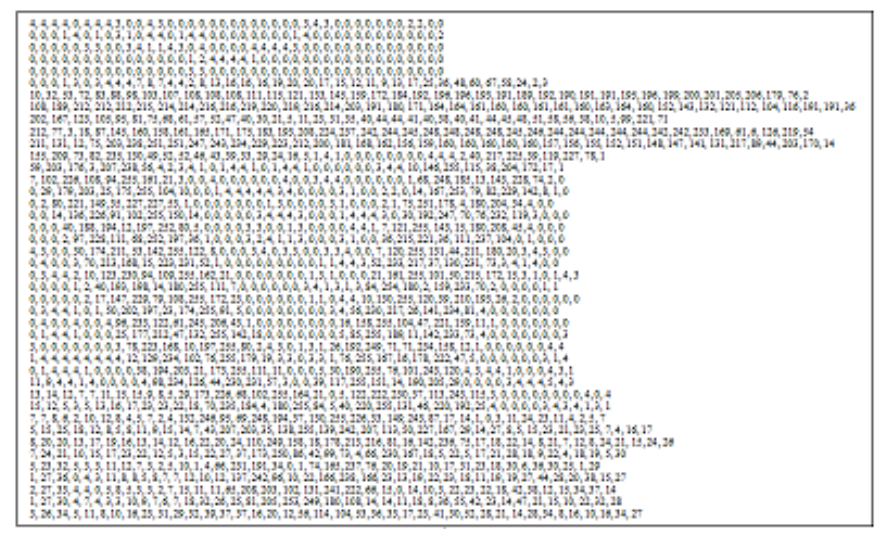

Figure 4. Test-data matrix. 


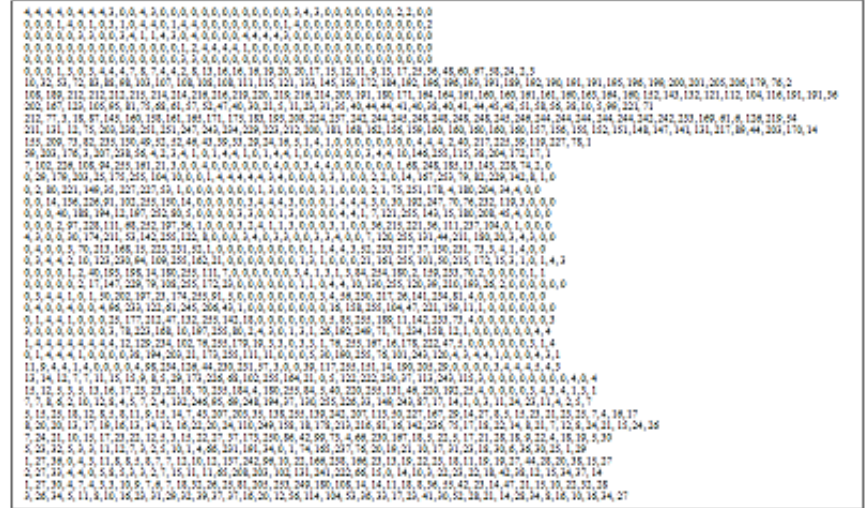

Figure 5. Train-data matrix.

TABLE 1

Normalized Euclidean Distance Result

\begin{tabular}{|c|c|c|c|}
\hline No & Data & $\begin{array}{c}\text { Euclidean } \\
\text { distance }\end{array}$ & $\begin{array}{c}\text { Recognized } \\
\text { as }\end{array}$ \\
\hline 1 & Data 1 & 0,0819 & Sign \\
\hline 2 & Data 2 & 0,0326 & Sign \\
\hline 3 & Data 3 & 0,0424 & Sign \\
\hline 4 & Data 4 & 0,0913 & Sign \\
\hline 5 & Data 5 & 0,0377 & Sign \\
\hline 6 & Data 6 & 0,1880 & Sign \\
\hline 7 & Data 7 & 0,1451 & Sign \\
\hline 8 & Data 8 & 0,3848 & Sign \\
\hline
\end{tabular}

Table 1 shows results of normalized Euclidean distance of all data. The normalized Euclidean distance values in range of 0.0 and 2.0. It shows both data have slight different, but still recognized as a sign. From the whole system examination, it resulted a accuracy value of 0,65 . This value shows that the data was correctly classified by $65 \%$. The precision value has an outcome of 0,64 , shows that the exact result of the classification is $64 \%$. The recall value has an outcome of 0,94 . This value shows that the success rate of recognizing a data from the whole data is $94 \%$.

\section{CONCLUSSION}

This study has demonstrated that the application of Canny edge detection performs clear of edge detection results. The saliency map model of traffic signs detection has well generated with Canny edge detection and produce great sign identification. From the whole system examination, it resulted a accuracy value of 0,65 . This value shows that the data was correctly classified by $65 \%$. The precision value has an outcome of 0,64 , shows that the exact result of the classification is $64 \%$. The recall value has an outcome of
0,94. This value shows that the success rate of recognizing a data from the whole data is $94 \%$.

\section{REFERENCES}

[1] Bascón, Saturnino Maldonado. 2007. Road-Sign Detection and Recognition Based on Support Vector Machines. IEEE Transactions On Intelligent Transportation Systems, Vol. 8, No. 2, June 2007.

[2] Borji, Ali dan Laurent Itti. 2013. State-of-the-Art in Visual Attention Modeling. IEEE Transactions On Pattern Analysis And Machine Intelligence, Vol. 35, No. 1, January 2013.

[3] Gao, Shangbing dan Yunyang Yan. 2012. Salieny and Active Contour based Traffic Sign Detection. ISSN 1746-7659, England, UK Journal of Information and Computing Science Vol. 7, No. 3, 2012, pp. 235240 .

[4] Hechri, Ahmed dan Abdellatif Mtibaa. 2011. Lanes and Road Signs Recognition for Driver Assistance System. IJCSI International Journal of Computer Science Issues, Vol. 8, Issue 6, No 1, November 2011.

[5] Itti, L., Koch, C. dan Niebur, E. 1998. A Model Of Saliency-based Visual Attention For Rapid Scene Analysis. IEEE Transactions On Pattern Analysis And Machine Intelligence Volume 20, Issue 11, Nov 1998

[6] Itti, L. 2004. Automatic Visual Salience. Scholarpedia, 2(9):3327, 2007.

[7] Jayachandra, Chinni. dan H.Venkateswara Reddy. 2013. Iris Recognition based on Pupil using Canny edge detection and K-Means Algorithm. International Journal Of Engineering And Computer Science ISSN:2319-7242 Volume 2 Issue 1 Jan Page No. 221-225.

[8] Loy, Gareth. 2004. Fast Shape-based Road Sign Detection for a Driver Assistance System. Computer Vision andActive Perception Laboratory Royal Institute of Technology (KTH).

[9] Menteri Perhubungan RI. 2004. Rambu Lalu Lintas.

[10] Neibur, Ernst. 2007. Saliency Map.

[11] Parikh,N, L Itti, dan J. Weiland. 2010. Saliency-Based Image Processing ForRetinal Prostheses . Journal Of Neural Engineering J. Neural Eng. 7 (2010) 016006 (10pp).

[12] Polatsek, Bc. Patrik. 2015. Spatiotemporal Saliency Model Of Human Attention In Video Sequences (Thesis).

[13] Powers, David M W. 2011. Evaluation: From Precision, Recall and F-Factor to ROC, Informedness, Markedness \& Correlation. Journal of Machine Learning Technologies (2011) 2 (1): 37-63 [online].

[14] Putra, Dharma. 2010. Pengolahan Citra Digital. Yogyakarta: Andi.

[15] Putra, Dharma. 2009. Sistem Biometrika. Yogyakarta: Andi.

[16] Rambe, SJ. 2011. Bab 2 Landasan Teori Citra Digital.

[17] Treisman, Anne M. dan Garry Gelade. 1980. A Feature-Integration Theory Of Attention. Cognitive Psychology, vol. 12, pp. 97-136, 1980.

[18] Won,Woong-Jae, Sungmoon Jeong, dan Minho Lee. 2007. Road Traffic Sign Saliency Map Model. Proceedings of Image and Vision Computing New Zealand 2007, pp. 91-96, Hamilton, New Zealand.

[19] Zhang, Jianming dan Stan Sclaroff . 2013. Saliency Detection: A Boolean Map Approach. Journal IEEE International Conference on Computer Vision (ICCV). 